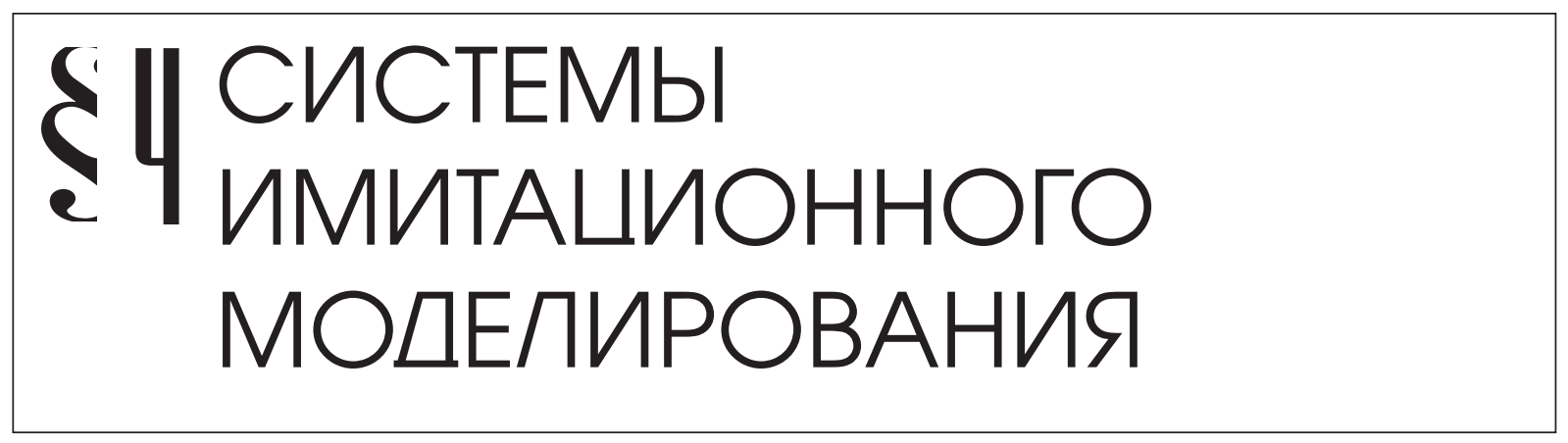

Кондратьев В. С.

\title{
ИМИТАЦИОННОЕ МОДЕЛИРОВАНИЕ ПРОЦЕССА УПРАВЛЕНИЯ СОСТОЯНИЕМ НАЛОГО-ОБЛАГАЕМОЙ БАЗЫ АДМИНИСТРАТИВНОЙ ЕДИНИЦЫ
}

\begin{abstract}
Аннотация: Предметом исследования движения налогооблагаемой базы (НОБ) административной единицы являются методы построения Имитационных моделей процесса управления состоянием НОБ как объекта в составе административной единицы и возможности имитационного моделирования движения НОБ, в целях её познания в условиях дальнейшего усложнения рыночных отношений как между предприятиями Административной Единицы (АE), так и между её администрацией и отдельным предприятием. Информационная технология управления состоянием НОБ в своих имитационных моделях являющихся инструментом в её составе необходима для административной единицы власти и состоит из совокупности приёмов, способов и средств, обеспечивающих ей оптимальное распределение ресурсов в процессе придания реальной НОБ желаемого состояния. В основу исследования движения НОБ как реального объекта в составе административной единицы положена методология управления состоянием любого объекта, рассматриваемого в любой предметной области, представленная в форме структурной модели логической организации методов и средств: - наблюдения параметров движения объекта; измерения значения хотя бы одного параметра движения объекта; моделирования движения объекта; оченки параметров движения объекта;выбора альтернативы и принятия решения; формирования из располагаемого ресурса управляющего воздействия на процесс движения объекта. Научная новизна предлагаемого инструмента в форме имитационной модели заключается в новой логической организации ( т.е. прочессе установления новых логических связей) методов и средств, входящих в состав процесса управления таким специфическим объектом как процесс движения НОБ, протекающий под действием конкурирующих субъектов внешней среды, систем и технологий из состава инфраструктур города и района. Преимущества предлагаемой Имитационной модели процесса управления состоянием НОБ в составе АЕ заключаются в возможности успешного решения проблемы расширения НОБ уже сегодня, т.к в её решении нуждает-
\end{abstract}


ся власть любого посёлка, города, района и региона. Инструментом, позволяющим властям административной единицы получить знания о вариантах решения задачи расширения НОБ, является представленная выше «Статистическая Имитационная Динамическая (СИД) модель процесса управления НОБ».В основу программно-математического обеспечения «системы поддержки принятия решений», входящей в состав «Статистической Имитачионной Динамической (СИД) модели процесса управления НОБ», могут быть положены алгоритмы, использующие методы статистических решений , теории игр, нечётких множеств и ряд методов динамического программирования. Ключевые слова: налогооблагаемая база, административная единица, имитационная модель, статистическая динамика, модель движения НОБ, прочесс принятия решения, anпаратно-программный комплекс, бескоолиционная игра, стратегия игрока, решение игры

\section{Введение}

Административные единицы власти, например, районов Московской области и их подразделения из состава систем управления налогооблагаемой базой (НОБ), функционируя в условиях рынка и сами, находясь в его составе, испытывают при определённых условиях трудности в выборе наилучшего управления состоянием НОБ близкого к её желаемому состоянию. На период 2013г-2014 г в большинстве районов Московской области, из налоговой облагаемой базы выпали физические лица, работающие в г. Москве и пополняющие бюджет этого города, а не своего района. Поэтому актуальность рассмотрения возникшего противоречия - проблемы расширения НОБ определяется необходимостью создания дополнительных рабочих мест в административной единице с целью привлечения на эти рабочие места специалистов, работающих в г. Москве, но проживающих на территории административной единицы.

Например, в 2012г по сведениям ГУ центра занятости населения Балашихинского района на предприятиях г. Москвы работали 42562 специалиста 34 профессий, что составляет $18 \%$ от общего количества работников района.

Рассмотрим более подробно вопросы управления НОБ. При этом под составом НОБ будем понимать совокупность предприятий всех сфер деятельности административной единицы и их работников, способных платить налоги с результатов своей деятельности.

Для властей административной единицы всегда имеют место две основные задачи: задача повышения объёма налоговых сборов в существующей базе;

задача создания рабочих мест для привлечения специалистов работающих в

г. Москве, но проживающих на территории административной единицы, расширяющих НОБ .

Управление не как менеджеры власти $A E$, а как процесс придания объекту - НОБ желаемого состояния - сложный многоэтапный процесс. В этом процессе руководителю административной единицы, желающему максимально пополнить её бюджет, при решении первой задачи, приходится сталкиваться как с фактами уклонения участников НОБ от уплаты налогов, так и с наличием не рентабельных предприятий. Избежать многих оши- 
бок в этом сложном процессе помогут излагаемые ниже результаты исследования возможности расширения НОБ административной единицы на имитационных моделях процесса управления состоянием НОБ.

Систематизированные знания о процессе управления состоянием НОБ , как впрочем и состоянием любого объекта, определяются знанием методологии управления состоянием объекта [1, 5].

\section{1. Методология управления движением ресурса - объекта}

Известно [4 ], что методология - совокупность методов и средств достижения цели логически организованных в учение в той или иной сфере деятельности человека. Как всякое учение методология управления состоянием объекта, например, производственным и экономическим процессами, процессом формирования НОБ и др. базируется на ряде положений.

Из логики процесса управления состоянием любого объекта, рассматриваемого в любой предметной области, следует, что к основным положениям методологии управления состоянием НОБ согласно структурной модели рис. 1 можно отнести:

1. наличие объекта - движение ресурса (в нашем случае - процесса формирования НОБ) и его желаемого состояния или цели управления;

2. наличие хотя бы одного метода и средства наблюдения параметров движения объекта;

3. наличие метода измерения значения хотя бы одного параметра движения объекта;

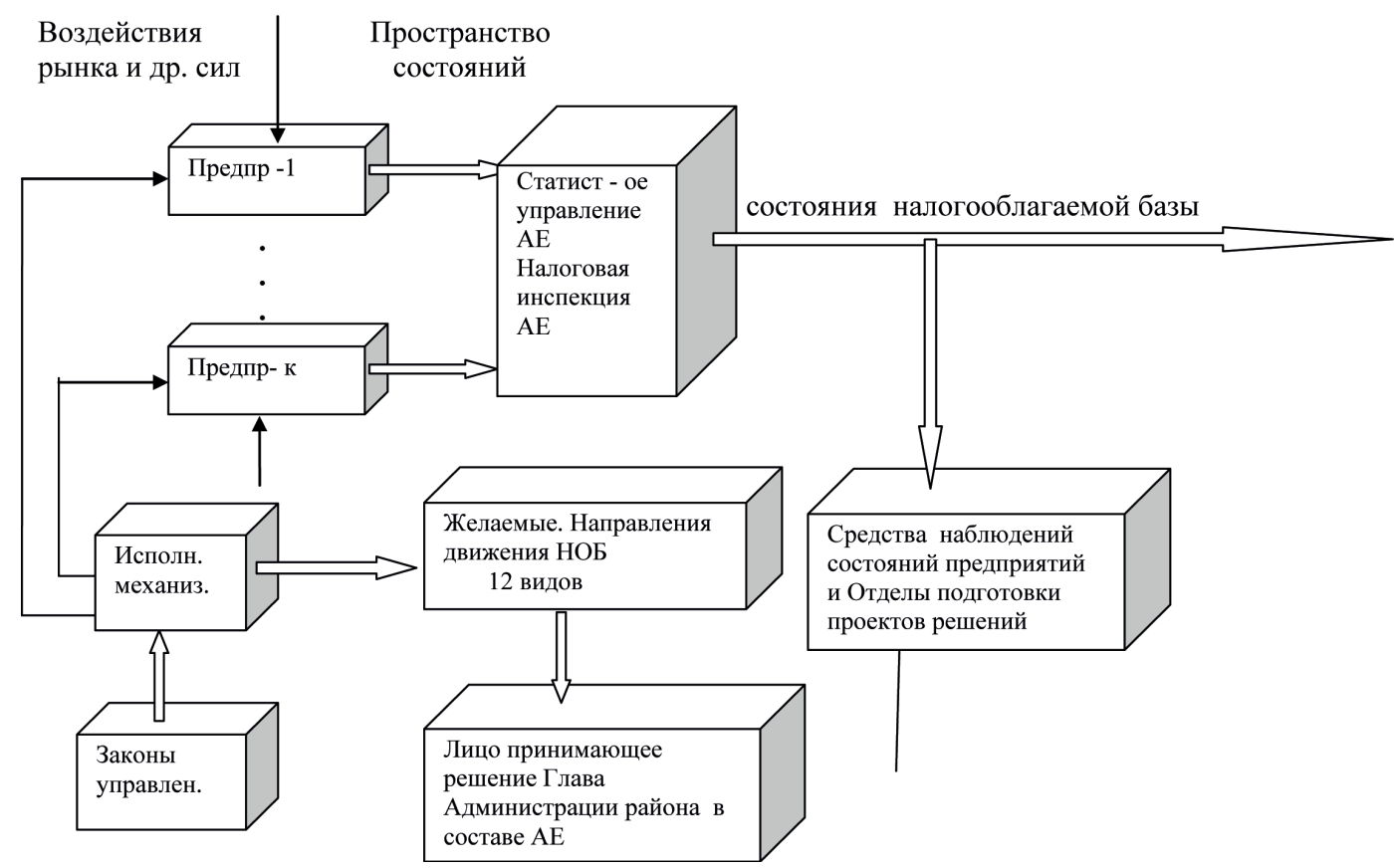

Рис.1 Структурная модель организации процесса управления движением НОБ и связей между методами её определяющими. 
4. наличие хотя бы одной модели движения объекта;

5. наличие хотя бы одного метода моделирования движений объекта;

6. наличие хотя бы одного метода оценки параметров движения объекта и выбора альтернативы из полученных значений оценок параметров движения объекта;

7. наличие хотя бы одного метода принятия решения при выборе альтернативы; 8. наличие хотя бы одного метода формирования из располагаемого ресурса управляющего воздействия на процесс движения объекта;

9. наличие логической организации (механизма согласования) всех имеющихся методов в учение в форме предметно-независимой методологии управления состоянием движения любого объекта из любой предметной области.

Из перечисленных выше положений необходимо выделить положение №9, как финишное положение, требующее наличия логической организации всех методов в учение, т.е. в методологию.

Для объективной оценки последствий от принятых решений, необходимо использовать современные достижения [1-6] в области информационных технологий. Здесь предлагается специальный инструмент управления состоянием НОБ, построенный на базе изложенной выше методологии и реализованный в компьютерном АПК. Инструмент позволяет в течение 1.5 - 2 -х часов выбрать оптимальное решение и оценить его последствия на каждом временном отрезке реализации каждого этапа. В составе инструмента имеется статистическая имитационная динамическая (СИД) модель процесса управления состоянием НОБ.

Модель имитирует последовательность реальных действий административной власти направленных, с одной стороны на выявление в составе НОБ, предприятий допускающих исакажения в статистике, не рентабельных предприятий и налогоплательщиков, допускающих уход от уплаты, а с другой стороны, на расширение объёма НОБ. Следует отметить, что современные НОБ в своём составе имеют от сотен до нескольких тысяч предприятий различной формы собственности, которые своей деятельностью создают многопрофильный продукт, являющийся основной компонентой НОБ.

Предприятия различных форм собственности и их работники представляют собой исполнителей законов управления сформированных управляющими из состава административной единицы, каждый из которых имеет свой локальный объект, входящий в состав НОБ. Предприятия из состава НОБ испытывают как внешние, так и внутренние воздействия, носящие различный характер имеющие место, время, направление и силу приложения .

Структурная модель организации процесса управления состоянием НОБ содержит предприятия, осуществляющие налоговые выплаты в реальном масштабе времени, которые отображаются в налоговой инспекции, формируя реальный процесс движения НОБ во времени и пространстве. Зафиксированные на конкретный момент времени параметры движения НОБ определяют её конкретное состояние, которое лицо принимающее решение (ЛПР) после оценки в модели сравнивает с желаемым состоянием НОБ. Если противоречия велики и не соответствуют требования ЛПР, то формируются законы управления и соответствующие им управляющие сигналы поступают к конкретным исполнителям из состава « исполнительного механизма», под которым понимается механистическое отображение взаимоотношений между исполнителями.

Под действием законов управления исполнители из состава предприятий расширяют 
ассортимент производимой продукции, увеличивают количество рабочих мест и реализуют ряд других мероприятий, придавая НОБ желаемое состояние.

Из приведенного выше описания логики работы структурной модели процесса управления состоянием НОБ можно создать «Статистическую имитационную динамическую модель» процесса управления НОБ и реализовать её в компьютерном аппаратно-программном комплексе (АПК), разбив его программное обеспечение на следующие ниже блоки представленные на рис.№2

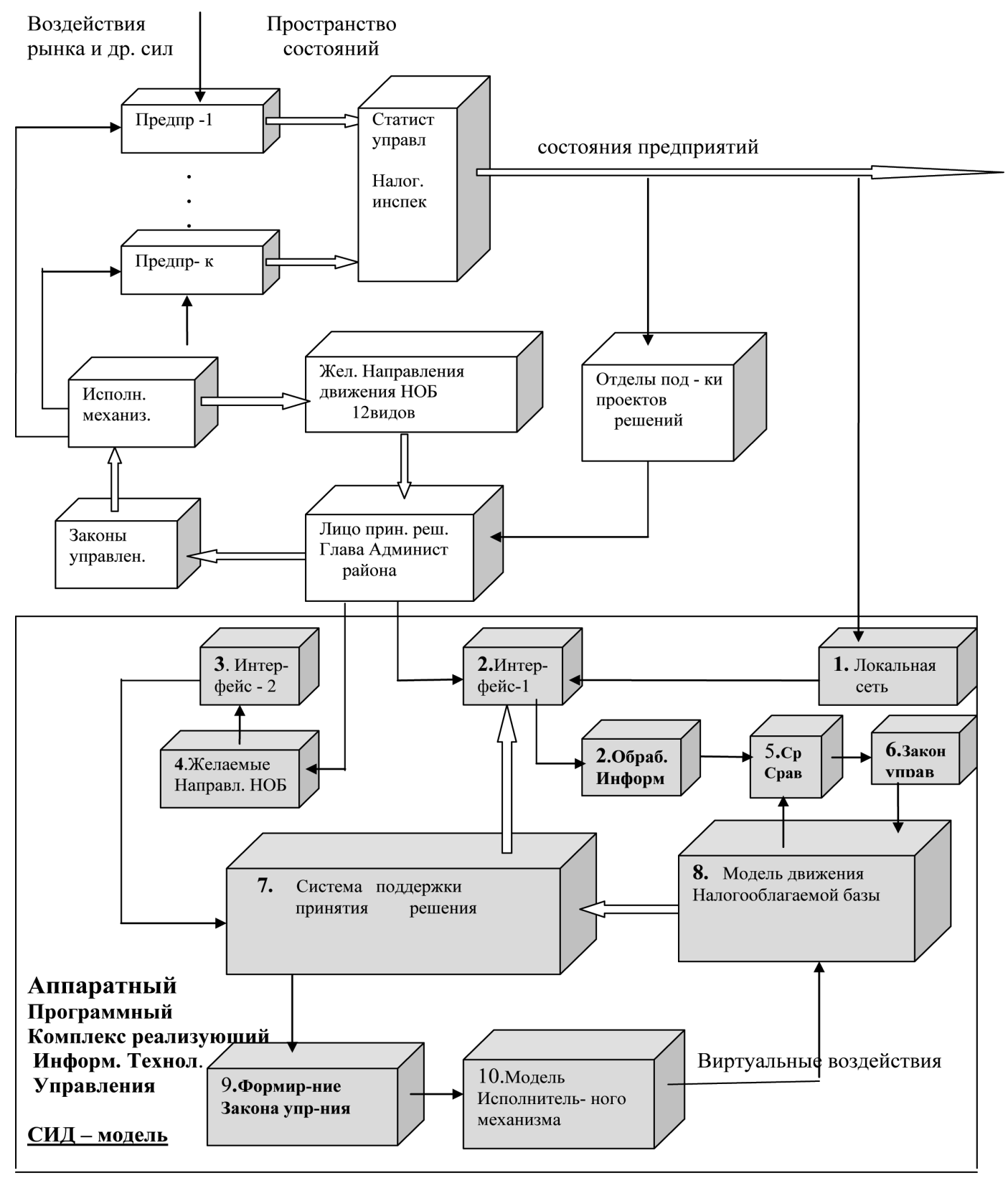

Рис № 2 Статистическая имитационная динамическая модель организации процесса управления НОБ и её реализация в компьютерном аппаратно-программном комплексе (АПК). 
1. блок обработки входного потока данных, поступающих из локальной сети;

2. интерфейс - 1;

3. интерфейс - 2 ;

4. блок формирования желаемых значений параметров НОБ по каждому направлению. утверждённому советом по бюджету при главе района;

5. сравнения оцененных в модели значений параметров НОБ с реальными параметрами;

6. блок формирования законов управления состоянием модели движения НОБ;

7. блок Лица Принимающего решения (ЛПР) с системой поддержки принятия решений;

8. модель процесса движения НОБ;

9. блок формирования законов управления значениями параметров НОБ;

10. модель функционирования «исполнительного механизма» по формированию виртуальных воздействий на модель движения значений параметров HOБ.

\section{2. Функционирование имитационной модели управления состоянием НОБ в информационной технологии}

Десять блоков, входящих в состав « Статистической имитационной динамической» модели процесса управления НОБ» представляют ИНСТРУМЕНТ, реализующий информационную технологию управления состоянием НОБ и позволяющий преобразовывать информационный ресурс реального процесса управления в информацию в форме оценок последствий от принятого решения.

Десять блоков реализующих соответствующие методы, связанные между собой логикой процесса управления, отражают процесс преобразования его информационного ресурса в информацию о возможных направлениях движения управляемого объекта и образуют ИНСТРУМЕНТ, содержащий виртуальную модель реального процесса управления состоянием НОБ.

Функционирование ИНСТРУМЕНТА складывается из нескольких этапов.

На первом этапе - с выхода локальной сети поток данных о состоянии НОБ каждого предприятия из списка и общей НОБ физических лиц, сформированных в «Статистическом управлении» Административной Единицы, поступает на вход блока обработки информации, в котором производится преобразование потока данных в пространство параметров НОБ и на вход блока «интерфейс-1». Лицо принимающее решение имеет возможность наблюдать на экране дисплея ЭВМ как текущее состояние каждого промышленного предприятия, так и текущее состояние НОБ как результат обработки потока данных по предприятиям преобразованный в обобщённые параметры НОБ. По результатам наблюдения состояний, указанных выше объектов, ЛПР переходит к реализации второго этапа. 
Примечание. Поскольку предприятия подают сведения о своём состоянии ежемесячно, а статистическая модель блока 8 отражает статистику развития предприятия за несколько предыдущих лет, то все параметры предприятия имеют среднее значение. Отклонения от него становятся чувствительными к любым искусственно введённым искажениям параметров (т.е. к обману, припискам, двойной бухгалтерии, уходу от налогов и т.п.).

На втором этапе - ЛПР выбирает, из утверждённого советом НОБ значения бюджета как управляющего ресурса, желаемое значения движения НОБ и значения параметров, характеризующих её состояние, задаёт их в ЭВМ, наблюдая результат на экране дисплея «интерфейса -2».

На третьем этапе - заданные ЛПР значения параметров состояния НОБ в выбранном направлении поступают в блок 7 - Систему Поддержки Принятия Решений ( СППР ). В этой системе производиться сравнение совокупности альтернатив, сформированных на выходе модели оценки параметров 8. движения НОБ в выбранном ЛРП направлении с желаемыми для него значениями параметров движения.

На четвёртом этапе - в СППР производятся поиск наиболее близкой альтернативы к желаемому значению по естественному критерию « минимального расхождения желаемого значения с альтернативой, предъявленной моделью 8.».

Поиск производится путём формирования в блоке 9. « Формирования закона управления» такого управляющего закона, который позволил бы в модели 10. «исполнительного механизма» сформировать виртуальное воздействие на модель движения НОБ, и тем самым получить на выходе модели 8. движения НОБ значение альтернативы более близкой к желаемому значению параметра НОБ.

По окончанию процедуры поиска, наилучшая альтернатива с выхода модели движения НОБ перешедшая в ранг решения, поступает в «интерфес - 1» и ЛПР на экране дисплея ЭВМ видит новые параметры состояния НОБ.

На пятом последнем этапе - если предложенный вариант состояния НОБ устраивает ЛПР, то оно вызывает через «интерфейс-1» рекомендации по воздействиям, которые привели НОБ в такое состояние и подключает реальных исполнителей для их реализации, в противном случае, производится дальнейший итерационный процесс, в котором повторяются все пять этапов поиска наилучшего состояния НОБ по назначенному критерию.

( например, максимуму прибыли от вложенного административного ресурса или максимум объёма собираемых налогов и т.п.).

Основным блоком в модели является блок, обеспечивающий ЛПР возможными вариантами решений, которые реализуются «Системой поддержки принятия решений».

Блок 7. «Система поддержки принятия решений» в своих программах реализует практически все возможные варианты, возникающие при движении НОБ под действием внешних и внутренних сил. При этом возможные варианты движений в процессе формирования НОБ подвергаются оцениванию в модели 8. процесса движения НОБ, представленной отдельным блоком. 


\section{3. Технология преобразования информационного ресурса реального процесса принятия решений в информацию, желаемую для ЛПР в его виртуальной модели}

Алгоритмы, используемые для формирования вариантов решений в блоке «Система поддержки принятия решений» рис.№1 как вариант технологии строятся на основе методов:

- линейного, нелинейного и динамического программирования;

- статистических решений;

- теори игр различного рода;

- нечётких множеств и ряде других методов.

Конечно, в рамках этой работы не представляется возможным рассмотреть все перечисленные выше методы, но наиболее важный из них: - метод бескоалиционной игры двух игроков, применительно к поставленной задаче исследования возможности расширения НОБ, рассмотреть необходимо.

В конечной бескоалиционной игре двух игроков (КБИДИ), например, инвестора

( игрок 1) и главы административной единицы (игрок 2) по расширению НОБ, каждый из них на переговорах делает ряд ходов: - выбирает одну стратегию из имеющегося у него конечного числа стратегий, и после этого он получает свой выигрыш, согласно определённым для каждого из них матрицам выигрышей. Другими словами КБИДИ полностью определяется двумя матрицами выигрышей для двух игроков. Поэтому такие игры часто называются [6]биматричными.

Изложим кратко и в общем виде алгоритм формирования решений в блоке 7 рис.2 «Система поддержки принятия решений».

Пусть у игрока 1 имеется $m$ стратегий, $i=\overline{1, m}$, у игрока 2 имеется $n$ стратегий, $j=\overline{1, n}$. Выигрыши игроков 1 и 2 соответственно задаются матрицами

$$
A=\left(\begin{array}{ccc}
a_{11} & \cdots & a_{1 n} \\
\vdots & & \\
a_{m 1} & \cdots & a_{m n}
\end{array}\right), \quad B=\left(\begin{array}{ccc}
b_{11} & \cdots & b_{1 n} \\
\vdots & & \\
b_{m 1} & \cdots & b_{m n}
\end{array}\right)
$$

Будем считать полный набор вероятностей $x=(x 1, \ldots, x m)$ применения игроком 1 своих чистых стратегий смешанной стратегией игрока 1, и $y=(y 1, \ldots, y n)$ - смешанной стратегией игрока 2. тогда средние выигрыши игроков 1 и 2 соответственно равны

$$
\left\{\begin{array}{l}
E(A, x, y)=\sum_{i=1}^{\mathrm{m}} \sum_{j=1}^{n} \mathrm{a}_{i j} x_{i} y_{j}=\mathrm{xAy}^{\mathrm{T}} \\
E(B, x, y)=\sum_{i=1}^{\mathrm{m}} \sum_{j=1}^{n} \mathrm{~b}_{i j} x_{i} y_{j}=\mathrm{xBy}^{\mathrm{T}}
\end{array}\right.
$$

Ситуация равновесия для биматричной игры составляет пару (x,y) таких смешанных 
стратегий игроков 1 и 2, которые удовлетворяют неравенствам :

$$
\begin{cases}\sum_{j=1}^{n} \mathrm{a}_{i j} y_{j} \leq \sum_{i=1}^{\mathrm{m}} \sum_{j=1}^{n} \mathrm{a}_{i j} X_{i} y_{j} \quad(\mathrm{i}=\overline{1, \mathrm{~m}}) \\ \sum_{i=1}^{m} \mathrm{~b}_{i j} X_{i} \leq \sum_{i=1}^{\mathrm{m}} \sum_{j=1}^{n} \mathrm{~b}_{i j} X_{i} y_{j} \quad(\mathrm{j}=\overline{1, \mathrm{n}})\end{cases}
$$

или

$$
\left\{\begin{array}{lll}
A y^{T} \leq \mathrm{xAy}^{\mathrm{T}} & \left(=E_{1}(A, x, y)\right) & \underline{\underline{\left(1^{\prime}\right)}} \\
x B \leq \mathrm{xBy}^{\mathrm{T}} & \left(=E_{2}(B, x, y)\right) & \underline{\underline{\left(2^{\prime}\right)}}
\end{array}\right.
$$

Для определения ситуаций равновесия необходимо решить систему неравенств (1) и (2) ((1') и (2')) относительно неизвестных $x=(x 1, \ldots, x m)$ и $y=(y 1, \ldots, y n)$ при условиях

$$
\sum_{i=1}^{m} x_{i}=1, \quad \sum_{j=1}^{n} y_{j}=1, \quad x_{i} \geq 0 \quad(i=\overline{1, m}), \quad y_{j} \geq 0 \quad(j=\overline{1, n}) .
$$

Известно [4,6],что каждая биматричная игра имеет по крайней мере одну ситуацию равновесия.

Содержательно это означает, что решения системы (1) и (2) включают для игрока 1 множество $K$ - решений , а для игрока 2 множество $L$ - решений.

Множество $K$ решений системы (1) - (2) состоит из:

всех ситуаций вида $(0 ; y)$, если $a_{1} y-a_{2} \leq 0 ; 0 \leq y \leq 1$;

ситуаций вида $(x ; y)$, если $a_{1} y-a_{2}=0 ; 0<x<1$;

ситуаций вида $(1 ; y)$, если $a_{1} y-a_{2} \geq 0 ; 0 \leq y \leq 1$.

Множество L приемлемых для него ситуаций состоит из :

всех ситуаций вида $(x, 0)$, если $b_{1} x-b_{2}<0 ; 0 \leq x \leq 1$,

всех ситуаций вида $(x, y)$, если $b_{1} x-b_{2}=0 ; 0 \leq x \leq 1 ; 0<y<1$,

всех ситуаций вида $(x, 1)$, если $b_{1} x-b_{2}>0 ; 0 \leq x \leq 1$.

Решением игры является пересечение множеств $K$ и $L$, т.е. те значения $x$ и $y$, которые являются общими для множеств $K$ и $L$. Графически это можно представить в виде рис. 3

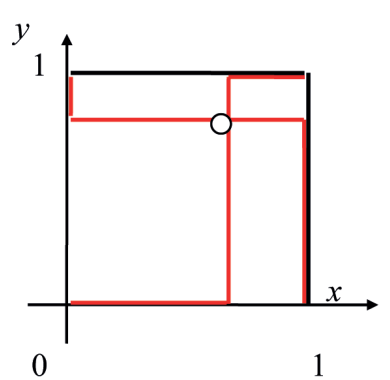

a)

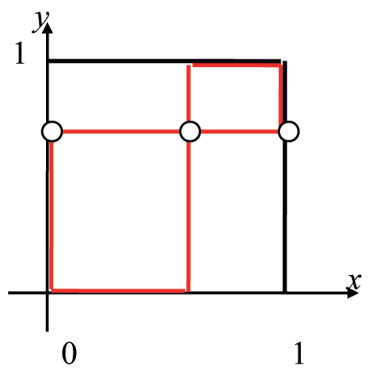

б)

рис.3 $($ а, 6$)$ 
При этом зигзаги $K$ и $L$ могут быть не только одинаковой, но и противоположной направленности. В первом случае зигзаги имеют одну точку пересечения, а во втором три. Средние выигрыши при этом определяются по формулам (*), если в них подставить полученное решение $x$ и $y$ (рис.2а).

Очевидно, $\alpha$ совпадает с оптимальной стратегией игрока 1 в матричной игре с матрицей $A$, а $\beta$ - с оптимальной стратегией игрока 2 в матричной игре с матрицей $\mathrm{B}$. Отсюда можно сделать вывод, что равновесная ситуация направляет поведение игроков не только на максимизацию своего выигрыша, сколько на минимизацию выигрыша противоположного игрока.

С другой стороны, естественно, также считать подходящим, поведение игроков в конечных бескоалиционных играх, направленное на максимизацию своего выигрыша с учётом максимального противодействия игрока. Будем считать: - подходящей стратегией игрока 1 его оптимальную смешанную стратегию м матричной игре с матрицей $A$;

$$
A=\left(\begin{array}{cc}
-10 & 2 \\
1 & -1
\end{array}\right), \quad B=\left(\begin{array}{cc}
5 & -2 \\
-1 & 1
\end{array}\right)
$$

(например: если игроки применяют свои первые стратегии, Инвестор решает строить 1 объект, а городские власти разрешают его постройку, тогда город получает выигрыш 5 млн. у.е и предприятие в составе НОБ и рабочие места , а Инвестор теряет 10 млн, и т.д.)

Решение в имитационной модели .

Для этой игры имеем :

$$
\begin{aligned}
& a_{1}=a_{11}-a_{12}-a_{21}+a_{22}=-10-2-1-1=-14<0, \\
& a_{2}=a_{22}-a_{12}=-1-2=-3, \\
& \alpha=\frac{a_{2}}{a_{1}}=\frac{-3}{-4}=\frac{3}{14} .
\end{aligned}
$$

Так как $a_{1}<0$, то множество решений $\mathrm{K}$ имеет следующий вид :

$(0, y)$ при $\frac{3}{14} \leq y \leq 1 ; \quad\left(x, \frac{3}{14}\right)$ при $0 \leq x \leq 1 ; \quad(1, y) \quad$ при $0 \leq y \leq \frac{3}{14}$.

Для 2 игрока имеем :

$$
\begin{aligned}
& b_{1}=b_{11}-b_{12}-b_{21}+b_{22}=5+2+1+1=9>0, \\
& b_{2}=b_{22}-b_{21}=1+1=2, \quad \beta=\frac{2}{9} .
\end{aligned}
$$


Так как $\mathrm{b}_{1}>0$, то множество решений $L$ имеет следующий вид :

$(x ; 0)$, при $0 \leq x \leq \frac{2}{9}$;

$\left(\frac{2}{9} ; y\right)$, при $0 \leq y \leq 1$;

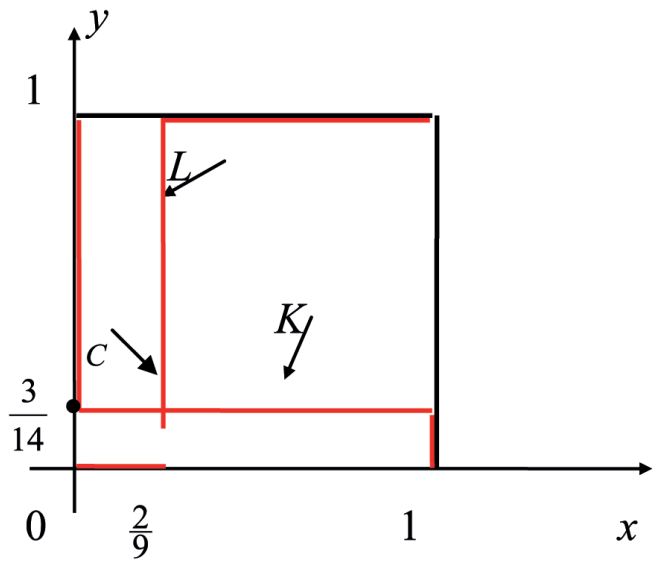

(x; 1), при $\frac{2}{9} \leq x \leq 1$.

Точка пересечения множеств $L$ и $K$ есть точка C с координатами $x=\frac{2}{9} ; y=\frac{3}{14}$ и отражает соответственно приемлемые стратегии Инвестора и города.

При этом выигрыш соответственно равен:

$$
\begin{aligned}
& E_{1}(A, x, y)=(x, 1-x)\left(\begin{array}{ll}
a_{11} & a_{12} \\
a_{21} & a_{22}
\end{array}\right)\left(\begin{array}{c}
y \\
1-y
\end{array}\right)=\left(\frac{2}{9} ; \frac{7}{9}\right)\left(\begin{array}{cc}
-10 & 2 \\
1 & -1
\end{array}\right)\left(\begin{array}{c}
3 / 14 \\
11 / 14
\end{array}\right)=-\frac{4}{7} \\
& E_{2}(A, x, y)=(x, 1-x)\left(\begin{array}{ll}
b_{11} & b_{12} \\
b_{21} & b_{22}
\end{array}\right)\left(\begin{array}{c}
y \\
1-y
\end{array}\right)=\frac{1}{3}
\end{aligned}
$$

Алгоритм, ведения переговоров по расширению налогооблагаемой базы административной единицы, изложенный выше в форме игры Инвестора с Главой административной единицы и представленный в блоке « Система поддержки принятия решений», при его реализации в компьютерном АПК, позволит игрокам принять эффективное решение и успешно управлять состоянием НОБ.

\section{Выводы:}

1. Преимущества предлагаемой Имитационной модели процесса управления состоянием НОБ в составе АЕ заключаются в возможности успешного решения проблемы расширения НОБ уже сегодня, т.к в её решении нуждается власть любого посёлка, города , района и региона.

2. Инструментом, позволяющим властям административной единицы получить знания о вариантах решения задачи расширения НОБ, является представленная выше «Статистическая Имитационная Динамическая (СИД) модель процесса управления НОБ».

3. В основу программно-математического обеспечения «системы поддержки приня- 
тия решений», входящей в состав «Статистической Имитационной Динамической (СИД) модели процесса управления НОБ», могут быть положены алгоритмы, использующие методы статистических решений, теории игр и нечётких множеств и ряд других методов динамического программирования

\section{Библиография :}

1. Кондратьев В.С. Эффективные системы менеджмента. Учебник М: Гамма Групп. 2013г. 410 стр.

2. Кондратьев В.С. Основы методологии управления экономическим состоянием предприятия. Сборник научных статей ИБПИТ. М О. г. Балашиха. : ИБПИТ 2004г.

3. Кондратьев В.С. Информационная технология управления состоянием промышленной политики административной единицы. Сборник научных статей МОИУ. М 0. г. Балашиха. : ИБПИТ 2005г.

4. Коршунов Ю.М. Математические основы кибернетики. М.: Энергоиздат, 1987г.

5. Кондратьев В.С. Методология управления движением ресурсов, реализуемая системами менеджмента. Информационные материалы Rassian-Balkan Innovation Forum Сербия Белград Тезисы 2010г. стр. 30-35

6. Гермейер Ю.Б. Игры с не противоположными интересами. М.: Наука, 1976г.

7. Коробейников А.Г., Гришенцев А.Ю., Кутузов И.М., Пирожникова 0.И., Соколов К.О., Литвинов Д.Ю. Разработка математической и имитационной моделей для расчета оценки защищенности объекта информатизации от несанкционированного физического проникновения // NB: Кибернетика и программирование. - 2014. - 5. - C. 14 - 25. DOI: 10.7256/2306-4196.2014.5.12889. URL: http://www.e-notabene.ru/kp/article_12889.html

8. Лабковская Р.Я., Козлов А.С., Пирожникова О.И., Коробейников А.Г. Моделирование динамики чувствительных элементов герконов систем управления // NB: Кибернетика и программирование. - 2014. - 5. - C. 70 - 77. DOI: 10.7256/2306-4196.2014.5.13309. URL: http://www.e-notabene.ru/kp/article_13309.html

9. Олзоева С.И. Метод автоматической классификации в распределенном моделировании систем // Программные системы и вычислительные методы. - 2013. - 2. - С. 160 - 163.

DOI: 10.7256/2305-6061.2013.2.7344.

10. Кульба В.В., Шульц В.Л., Шелков А.Б., Чернов И.В. Методы управления эффективностью реализации социально-экономических целевых программ // Тренды и управление. - 2013. - 4. - С. 4 - 28.

DOl: $10.7256 / 2307-9118.2013 .4 .9603$

\section{References:}

1. Kondrat'ev V.S. Effektivnye sistemy menedzhmenta. Uchebnik M: Gamma Grupp. 2013g. 410 str.

2. Kondrat'ev V.S. Osnovy metodologii upravleniya ekonomicheskim sostoyaniem predpriyatiya. Sbornik nauchnykh statei IBPIT. M 0. g. Balashikha. : IBPIT 2004g.

3. Kondrat'ev V.S. Informatsionnaya tekhnologiya upravleniya sostoyaniem promyshlennoi politiki administrativnoi edinitsy. Sbornik nauchnykh statei MOIU. M 0. g. Balashikha. : IBPIT 2005g.

4. Korshunov Yu.M. Matematicheskie osnovy kibernetiki. M.: Energoizdat, 1987g. 
5. Kondrat'ev V.S. Metodologiya upravleniya dvizheniem resursov, realizuemaya sistemami menedzhmenta. Informatsionnye materialy Rassian-Balkan Innovation Forum Serbiya Belgrad Tezisy 2010g. str. 30-35

6. Germeier Yu.B. Igry s ne protivopolozhnymi interesami. M.: Nauka,1976g.

7. Korobeinikov A.G., Grishentsev A.Yu., Kutuzov I.M., Pirozhnikova 0.I., Sokolov K.O., Litvinov D.Yu. Razrabotka matematicheskoi i imitatsionnoi modelei dlya rascheta otsenki zashchishchennosti ob"ekta informatizatsii ot nesanktsionirovannogo fizicheskogo proniknoveniya // NB: Kibernetika i programmirovanie. - 2014. - 5. - C. 14 - 25. DOI: 10.7256/2306-4196.2014.5.12889.URL: http://www.e-notabene.ru/kp/article_12889.html

8. Labkovskaya R.Ya., Kozlov A.S., Pirozhnikova 0.I., Korobeinikov A.G. Modelirovanie dinamiki chuvstvitel'nykh elementov gerkonov sistem upravleniya // NB: Kibernetika i programmirovanie. - 2014. - 5. - C. 70 - 77. DOI: 10.7256/2306-4196.2014.5.13309. URL: http://www.e-notabene.ru/kp/article_13309.html

9. Olzoeva S.I. Metod avtomaticheskoi klassifikatsii v raspredelennom modelirovanii sistem // Programmnye sistemy i vychislitel'nye metody. - 2013. - 2. - C. 160 - 163. D0I: 10.7256/2305-6061.2013.2.7344.

10. Kul'ba V.V., Shul'ts V.L., Shelkov A.B., Chernov I.V. Metody upravleniya effektivnost'yu realizatsii sotsial'no-ekonomicheskikh tselevykh programm//Trendy i upravlenie. -2013. - 4. - C. 4-28. D0l:10.7256/2307-9118.2013.4.9603. 